\title{
A graphene-based chemical nose/tongue approach for the identification of normal, cancerous and circulating tumor cells
}

\author{
Li Wu ${ }^{1,2}$, Haiwei Ji ${ }^{1,2}$, Yijia Guan ${ }^{1,2}$, Xiang $\operatorname{Ran}^{1,2}$, Jinsong $\operatorname{Ren}^{1}$ and Xiaogang $\mathrm{Qu}^{1}$
}

The development of reliable, cost-effective, and sensitive detection and monitoring assays is particularly important for the early diagnosis and treatment of cancer. Here, we report an array-based system for cell sensing using a 'chemical nose/tongue' approach that exploits subtle changes in the physicochemical nature of different cell surfaces. A family of synthetic and biomolecular functionalized graphene elements was used to develop the sensing array. Their different interactions with dissimilar cell surfaces were transduced to an ultrasensitive electrochemical impedance signal, providing a fingerprint for the classification and identification of different cells. This electrochemical array sensing platform has ultrahigh sensitivity that can detect a singlecell type from one cell. Furthermore, it can discern different cell types at levels as low as 100 cells with high accuracy for (i) different cell types; (ii) cancerous, multidrug-resistant cancerous and metastatic human breast cells; and (iii) artificial circulating tumor cells (CTCs). The graphene-based electrochemical sensing platform presented here can more generally be applied to array sensor fabrication and can provide highly efficient signal transduction pathways to improve the detection response of each recognition element.

NPG Asia Materials (2017) 9, e356; doi:10.1038/am.2017.11; published online 10 March 2017

\section{INTRODUCTION}

Cancer continues to be a major cause of mortality despite decades of treatment effort and expense. Drugs and surgery can be effective against early tumors, but they usually only briefly extend the survival of patients with metastatic cancer. Although much effort has been devoted to the development of efficacious therapeutics, there is still a need for improved methods for early detection. ${ }^{1}$ Early detection can significantly reduce cancer mortality. Death rates for some major types of cancers have declined recently because of the use of early detection methods. ${ }^{2}$ Thus, the development of reliable, cost-effective, and powerful detection and monitoring strategies for cancer is particularly important. Early cancer cell detection mainly focuses on the identification of cellular signatures because cancerous cells are differentiated from noncancerous cells on the basis of intracellular or extracellular (cell surface) biomarkers. ${ }^{3}$ In the past few decades, this field has made remarkable and substantial progress. Various promising detection methods involving the specific recognition of biomarkers on the cell surface (for example, overexpressed antigens) or intracellular biomarkers (for example, dysregulation of RNA/proteins inside the cell) have been developed, such as PCR, ${ }^{4}$ enzyme-linked immunosorbent assay, ${ }^{5}$ surface plasmon resonance, ${ }^{6}$ microcantilevers ${ }^{7}$ and bioimaging. ${ }^{8}$ However, the design and preparation of the specific ligand-based sensors are generally costly and time-consuming. Furthermore, these methods all require previous knowledge of specific biomarkers. Because cells do not always express unique biomarkers that allow for differentiation between tumors of a given type or their metastases, development in this field is severely restricted. For example, the detection of circulating tumor cells (CTCs) currently depends mainly on a single marker, EpCAM, the epithelial cell surface epitope. ${ }^{9}$ However, EpCAM is not expressed in some types of cancer cells, and whether the EpCAM-positive CTCs may or may not have any metastatic potential has been the subject of debate. More importantly, assays focused on epithelial markers might not provide any meaningful information on metastasis-initiating cells that arise from circulating cancer stem cells. ${ }^{10}$ Therefore, there is an urgent need to develop an efficient platform for cancer cell analysis, especially for CTCs. ${ }^{11,12}$ When this technology becomes clinically available, a blood sample may be used as a liquid biopsy in tumor diagnostics, which is easily accessible for both the patient and physician.

Array-based sensing approaches discriminate between analytes based on their overall signatures, affording an alternative to the specific recognition processes based on lock-and-key approaches. ${ }^{13,14}$ A distinct pattern of responses generated by the sensor array provides a fingerprint that allows for classification and identification of the analyte. This differential sensing method is analogous to mammalian olfaction, ${ }^{15}$ thus, it is termed a 'chemical nose/tongue' strategy and

\footnotetext{
${ }^{1}$ Laboratory of Chemical Biology and State Key laboratory of Rare Earth Resource Utilization, Changchun Institute of Applied Chemistry, Chinese Academy of Sciences, Jilin, China and ${ }^{2}$ University of Chinese Academy of Sciences, Beijing, China

Correspondence: Dr X Qu, Laboratory of Chemical Biology and State Key Laboratory of Rare Earth Resource Utilization, Changchun Institute of Applied Chemistry, Chinese Academy of Sciences, 5625 Renmin Street, Changchun, Jilin 130022, China.

E-mail: xqu@ciac.ac.cn

Received 18 March 2016; revised 13 August 2016; accepted 23 October 2016
} 
a

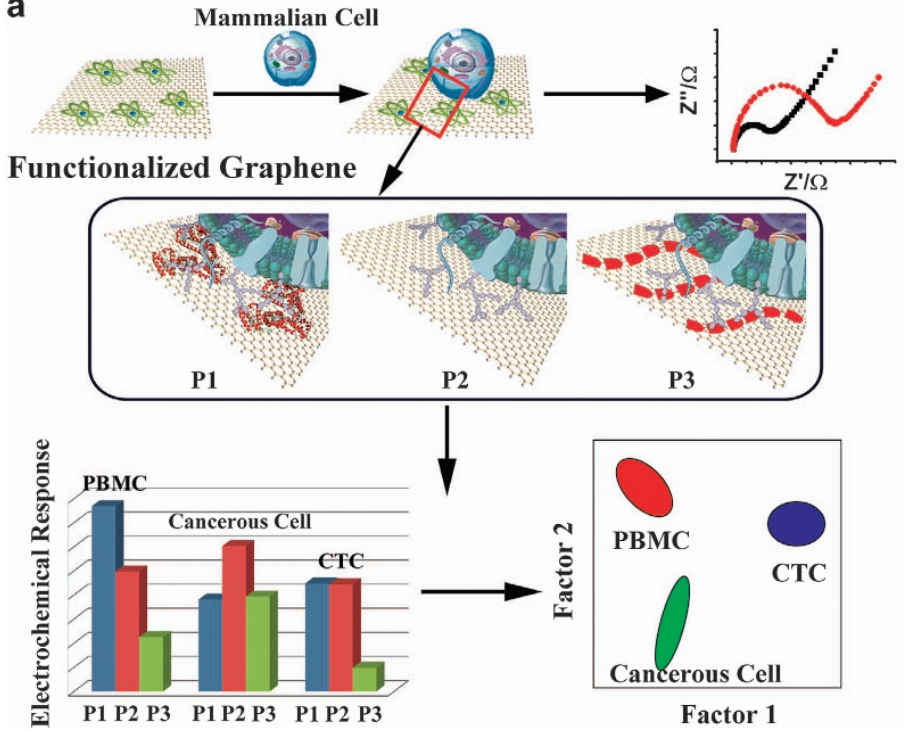

b

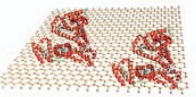

P1: BSA/CCG

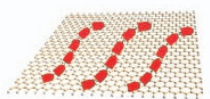

P3: Chit/CCG

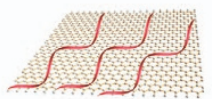

P5: DNA/CCG

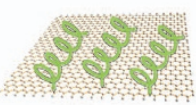

P7: PEG/CCG

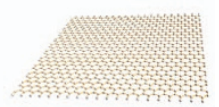

P2: CCG

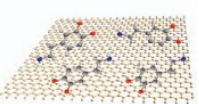

P4: DA/CCG

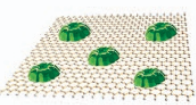

P6: Gel/CCG

Scheme 1 (a) Schematic presentation of the cell detection assay and the interactions between the functionalized graphene and the cell types; (b) Illustration of the functionalized graphene used in this study.

presents a powerful tool for discriminating subtly different analytes and their complex mixtures even in biomatrices. The potential of this method has been demonstrated in the detection of volatile agents, ${ }^{16}$ metal ions, ${ }^{17}$ amino acids, ${ }^{18}$ carbohydrates ${ }^{19}$ and proteins. ${ }^{20}$ Success has also been achieved in the identification of bacteria $^{21}$ and mammalian cells. ${ }^{22}$ Although promising, a key challenge of using this 'nose' strategy for early cancer diagnosis is the need to improve the sensitivity and discriminatory ability of the sensing approaches to detect subtle changes in analyte ratios, especially for CTC analysis with the interference of normal blood cells. A means of designing effective recognition elements and of developing a robust binding event-signal transduction platform is essential to boost the detection performance of these array sensors.

In this work, we use seven functionalized graphene probes (P1-P7, Scheme $1 b$ ) to provide an array-based electrochemical sensing system that can differentiate cancerous, multidrug-resistant cancerous and metastatic human breast cells, and can distinguish artificial CTC samples. Rather than using specific biomarker recognition, our method depends on differential affinity. The main advantage of our method is that the exact information about specific ligands and receptors is not required. We demonstrate that this syntheticbiomolecular sensor can discern different cell types at levels as low as 100 cells, and that the detection limit for the detection of a singlecell type is much lower $(\sim 1$ cell). Furthermore, taking the biomimetic interactions between the sensor array and the cell surface into account and using a sensitive electrochemical strategy, the sensing performance is instrumentally improved. We find that the combination of two random graphene probes mostly achieves distinct separation of the analytes, indicating that the graphene-based electrochemical method is promising for array-based sensor fabrication.

\section{MATERIALS AND METHODS}

\section{Electrochemical measurements}

Electrochemical impedance spectroscopy measurements were performed using a CHI 660B Electrochemistry Workstation (CHI, Austin, TX, USA) in $100 \mathrm{~mm}$ PBS $(\mathrm{pH}=7.5)$ containing a $10 \mathrm{~mm} \mathrm{~K}_{3}\left[\mathrm{Fe}(\mathrm{CN})_{6}\right] / \mathrm{K}_{4}\left[\mathrm{Fe}(\mathrm{CN})_{6}\right](1: 1)$ mixture with $1 \mathrm{M} \mathrm{KCl}$ as the supporting electrolyte. The impedance spectra were recorded within the frequency range of $10^{-2}-10^{5} \mathrm{~Hz}$, with a conventional three-electrode system comprising a platinum wire as the auxiliary electrode, $\mathrm{Ag} / \mathrm{AgCl}$ as the reference electrode, and modified glassy carbon electrodes (GCE) used as the working electrode. The amplitude of the applied sine wave potential in each case was $5 \mathrm{mV}$.

\section{Preparation of functionalized graphene}

Graphene oxide (GO) was synthesized from graphite using a modified Hummers method. ${ }^{23}$

Chemically converted graphene (CCG) was prepared following the procedures previously described with some modification. ${ }^{24}$ In brief, $2.5 \mathrm{ml}$ of GO $\left(1 \mathrm{mg} \mathrm{ml}^{-1}\right), 2 \mu \mathrm{l}$ of hydrazine solution, and $40 \mu \mathrm{l}$ of ammonia solution (25 $\mathrm{wt} \%$ in water) were mixed in a total volume of $10 \mathrm{ml}$. After being vigorously shaken or stirred for a few minutes, the vial was placed in a water bath $\left(\sim 95^{\circ} \mathrm{C}\right)$ for $1 \mathrm{~h}$. A graphene dispersion prepared according to the above procedure was used for further characterization and electrode modification in this work.

The nanocomposite BSA/CCG was synthesized according to a previous report. ${ }^{25}$ In brief, $50 \mu \mathrm{l}$ of GO $\left(1.0 \mathrm{mg} \mathrm{ml}^{-1}\right), 250 \mu \mathrm{l}$ of $\mathrm{H}_{2} \mathrm{O}$ and $200 \mu \mathrm{l}$ of $50 \mathrm{mg} \mathrm{ml}{ }^{-1}$ BSA were mixed. The $\mathrm{pH}$ of the mixture was rapidly raised to $\sim 12$ by adding $1.0 \mathrm{M} \mathrm{NaOH}$. The original yellow-brown solution of GO gradually turned black after heating at $90^{\circ} \mathrm{C}$ for $24 \mathrm{~h}$. The products were centrifuged at 12000 r.p.m., and the resulting black precipitates were redispersed in $\mathrm{ddH}_{2} \mathrm{O}$ (this process was repeated several times to ensure the successful removal of free, unbound BSA).

The chitosan-mediated graphene suspension (Chit/CCG) was prepared following previously described procedures. ${ }^{26}$ The GO suspension $(100 \mathrm{ml}$, $0.1 \mathrm{mg} \mathrm{ml}^{-1}$ ) was gradually added to an acidic aqueous solution of chitosan ( $100 \mathrm{ml}, 0.5 \mathrm{mg} \mathrm{ml}^{-1}$ in $0.5 \mathrm{M} \mathrm{HCl}$ ) while stirring to yield a uniform browncolored dispersion. L-ascorbic acid powder $(100 \mathrm{mg})$ was subsequently added to the above dispersion, and the mixture was held at $60^{\circ} \mathrm{C}$ for $6 \mathrm{~h}$ to reduce the GO to graphene.

Polydopamine-capped reduced GO (DA/CCG) was prepared as follows: ${ }^{27}$ $100 \mathrm{mg}$ of GO, $50 \mathrm{mg}$ of dopamine hydrochloride and $200 \mathrm{ml}$ of $10 \mathrm{~mm}$ Tris-Cl solution $(\mathrm{pH}=8.5)$ were mixed and dispersed by sonication for $10 \mathrm{~min}$ in an ice bath. The reaction mixture was stirred vigorously at $60^{\circ} \mathrm{C}$ for $24 \mathrm{~h}$. After completion of the reduction reaction, the product was collected by centrifugation, washed several times and redispersed in water.

ctDNA-functionalized graphene composites (DNA/CCG) were prepared according to our previous report. ${ }^{28}$ The ctDNA was heated at $95^{\circ} \mathrm{C}$ for $1-2 \mathrm{~h}$ 
to obtain single-stranded DNA. The GO dispersion $\left(10 \mathrm{ml}, 0.5 \mathrm{mg} \mathrm{ml}^{-1}\right)$ was mixed with single-stranded DNA $\left(10 \mathrm{ml}, 2 \mathrm{mg} \mathrm{ml}^{-1}\right)$, and hydrazine was added $(8 \mu \mathrm{l}, 85 \mathrm{wt} \%$; hydrazine/GO weight ratio $=7: 10)$; the mixture was refluxed at $10{ }^{\circ} \mathrm{C}$ for $1 \mathrm{~h}$. Then, the solution was centrifuged and washed several times with double-distilled water and dissolved in $10 \mathrm{ml}$ of water to obtain DNA/CCG.

Preparation of a gelatin reduced graphene nanocomposite (Gel/CCG): ${ }^{29}$ $1 \mathrm{~g}$ of gelatin was added to $50 \mathrm{ml}$ of water, followed by stirring for $0.5 \mathrm{~h}$ at $80^{\circ} \mathrm{C}$ for complete dissolution of the gelatin. Then, $50 \mathrm{ml}$ of $0.2 \mathrm{mg} \mathrm{ml}^{-1}$ $\mathrm{GO}$ aqueous dispersion was added dropwise into the homogeneous gelatin solution at $80^{\circ} \mathrm{C}$. After vigorously stirring for $30 \mathrm{~min}$, the mixture was stirred at $95^{\circ} \mathrm{C}$ for $24 \mathrm{~h}$ to allow the mixture to react. Finally, the resulting stable black dispersion was centrifuged at 17000 r.p.m. and washed several times with hot water to remove excess gelatin. The Gel/CCG obtained using this process was then redispersed in water before further use.

Fabrication of PEG functionalized graphene nanosheets (PEG/CCG) was performed as follows: a solution was prepared by vigorously stirring $20 \mathrm{mg}$ of GO, $100 \mathrm{mg}$ of amine-PEG 400 and $100 \mathrm{mg}$ of $\mathrm{KOH}$ in $50 \mathrm{ml}$ of $\mathrm{H}_{2} \mathrm{O}$ at $70^{\circ} \mathrm{C}$ for $24 \mathrm{~h} . \mathrm{NaBH}_{4}(10 \mathrm{ml}, 1 \mathrm{M})$ was added and the reaction was kept at $70^{\circ} \mathrm{C}$ for $2 \mathrm{~h}$. The solution was then centrifuged and washed several times with doubledistilled water and dissolved in $10 \mathrm{ml}$ of water.

\section{Cell culture and treatment}

PC-12 cells were cultured in flasks in Iscove's modified Dulbecco's medium (Gibco BRL, Grand Island, NY, USA) supplemented with 5\% fetal bovine serum and $10 \%$ horse serum in a humidified atmosphere containing $5 \% \mathrm{CO}_{2}$ at $37^{\circ} \mathrm{C}$. All the other cells used were grown in RPMI 1640 medium supplemented with $10 \%$ fetal bovine serum, streptomycin $\left(100 \mu \mathrm{g} \mathrm{ml}{ }^{-1}\right)$, and penicillin in a $5 \% \mathrm{CO}_{2}$-humidified chamber at $37^{\circ} \mathrm{C}$. After $72 \mathrm{~h}$ of culture, the cells were harvested by centrifugation for $5 \mathrm{~min}$ at 1000 r.p.m. and rinsed twice with PBS $(\mathrm{pH}=7.2)$. The precipitations obtained using this process were resuspended in the PBS solution to obtain a homogenous cell suspension. The cell number was determined using a Petroff-Hausser cell counter.

\section{Isolation of human peripheral blood mononuclear cells}

Human peripheral blood mononuclear cells (PBMCs) were isolated by FicollHypaque gradient centrifugation. ${ }^{30}$ Whole blood from healthy individual volunteers was collected in sterile heparinized vacutainer tubes. Equal volumes of blood and PBS were mixed gently with a sterile pipette in a 50-ml test tube. This blood-PBS mixture was slowly overlaid onto $15 \mathrm{ml}$ of Ficoll-Hypaque solution, and then centrifuged at 1500 r.p.m. for $40 \mathrm{~min}$ at $18^{\circ} \mathrm{C}$. The upper layer containing the plasma and platelets was removed with a sterile pipette. The buff-colored layer containing the PBMCs was transferred to a fresh tube, washed twice with $20 \mathrm{ml}$ PBS and resuspended in RPMI complete media. The cells were counted, and the viability of the cells was determined using a trypan blue exclusion test.

\section{Electrochemical array sensor for cell identification}

Before modification, 1.0, 0.3 and $0.05 \mu \mathrm{m}$ alumina (Buhler) was used to polish sequentially GCE $(\Phi=3 \mathrm{~mm}, \mathrm{CHI})$, followed by sonication of the electrodes for $3 \mathrm{~min}$. Functionalized graphene $(10 \mu \mathrm{l})$ was added dropwise onto the pretreated GCE and dried at room temperature. The electrochemical impedance spectroscopy of these graphene-modified electrodes was recorded. The surface was then washed with PBS buffer. Next, a $10 \mu \mathrm{l}$ suspension of cells at 100 cells was dropped onto the modified electrode surface and then incubated at $37^{\circ} \mathrm{C}$ for $30 \mathrm{~min}$. After carefully rinsing with PBS to remove non-captured cells, the electrodes were used for subsequent electrochemical impedance detection. The value of the electrochemical impedance before the addition of cells was subtracted from that obtained after the addition of cells to record the overall electrochemical response (Supplementary Tables S2-S6). Five replicates were obtained for all cell lines and the data were subjected to linear discriminant analysis (LDA). Because the interaction of the cells with the functionalized graphene array depends on the surface properties of the cell, each cell line possesses a unique electrochemical response with the functionalized graphene array. Therefore, for each cell line, we tested the electrochemical responses against 7 functionalized graphene probes 5 times, generating a $7 \times 5 \times 5$ matrix for (i) 5 different cancer cell lines: A549 (lung),
HeLa (cervical), HepG2 (liver), K562 (leukemia) and MCF-7 (breast), and (ii) 5 different cancer cell and normal cell lines NIH-3T3, PC-12, HEK-293T, MCF-7 and A549. The raw data were then subjected to jackknifed classification matrix analysis to reduce the size of the training matrix. The optimized data matrix was then processed using classical LDA. Similar procedures were also performed to identify the analytical samples (cancerous, multidrug-resistant cancerous and metastatic human breast cells; various concentrations of cancerous cells; artificial CTCs; and whole-blood samples). For the artificial CTC model, samples were prepared by spiking PBMCs with MCF-7 cells at specific ratios with an identical total cell number. The whole-blood samples were collected from three healthy donors and four breast cancer patients. For artificial CTC samples and whole-blood samples, a $10 \mu$ suspension of sample was added dropwise to the modified electrode surface and then incubated at $37^{\circ} \mathrm{C}$ for $30 \mathrm{~min}$ under a humid atmosphere. The prepared electrodes were rinsed carefully to remove the non-captured cells before being used for subsequent electrochemical impedance detection.

\section{RESULTS AND DISCUSSION}

\section{Design of the sensor array}

The cell membrane surface is mainly composed of integral membrane proteins, amphipathic phospholipids and carbohydrates, and their proportions are different in various cell types. The differences in cell membrane composition, such as charge and hydrophobicity, indicate the physiochemical diversities between cell types. Such physicochemical diversities could potentially be identified via an array-based 'chemical nose' approach that depends on the interactions between multiple reporter elements and the target cell. ${ }^{22}$ In view of this, we constructed a combinatorial electrochemical sensor array that consisted of sensors with synthetic and biomolecular functionalized graphene elements, which possessed different hydrophobic properties and various charges. These graphene-based sensors may have biomimetic interactions with cells, such as protein-protein surface interactions. As depicted in Scheme 1a, functionalized graphene nanocomposites, which are attractive electrode materials, were used to modify the GCEs as recognition elements. When mammalian cells are incubated with these modified electrodes, graphene nanocomposites are expected to interact with phospholipids, membrane proteins and carbohydrates of the cell surface through both electrostatic and hydrophobic interactions. These interactions are responsible for conjugating the target cells on the electrode surface, and subsequently, the binding event can be transduced to an ultrasensitive electrochemical impedance response. Since the graphene probes varied in charge, hydrophobicity and hydrophilicity, they contained chemical diversity to respond to dissimilar cell surfaces, serving as a sensor array featuring selective receptors. Accordingly, a unique global pattern produced from a set of sensors in the array provided a fingerprint that allowed for classification and identification of the cells.

\section{Materials characterization}

Graphene, a single atom-thick plane of carbon, has attracted much attention in recent years due to its extraordinary electronic, optical, magnetic, thermal and mechanical properties. ${ }^{31}$ Significant progress has been made in the development of biological applications using graphene and its derivatives, such as the creation of biosensors and nanocarriers for drugs and gene delivery and use in cell imaging and as phototherapy materials. ${ }^{32}$ As a super quencher, which can quench the fluorescence of virtually any fluorophore with ultrahigh efficiency, graphene is considered to be the superior candidate for chemical nose sensor array fabrication. ${ }^{33,34}$ Although the use of graphene in sensor arrays shows great promise, there have been few studies on sensor arrays and graphene-based array sensing remains to be explored. In the past, we have demonstrated that a graphene-based 

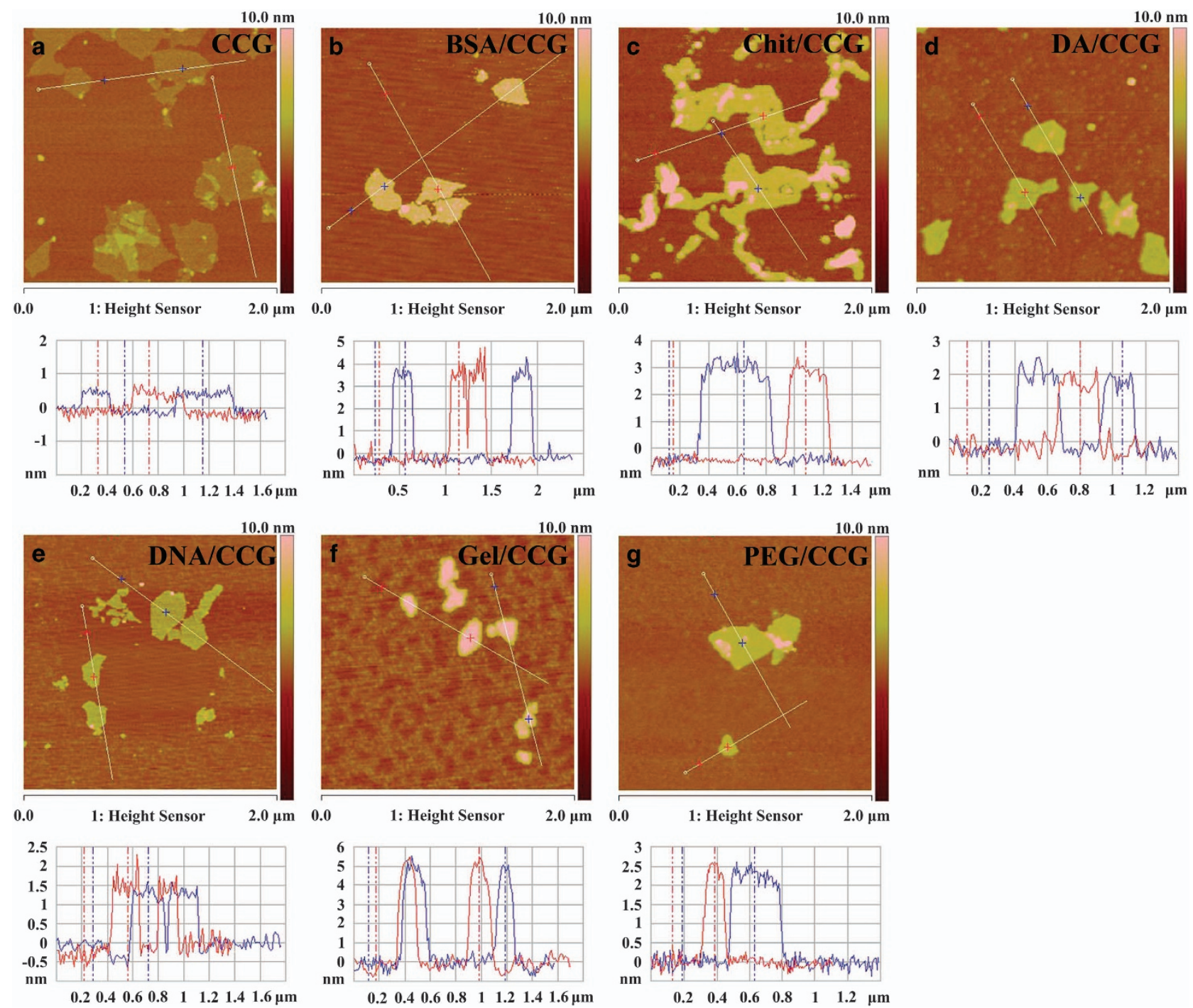

Figure 1 Representative atomic force microscopy (AFM) images of the as-synthesized functionalized graphene and their corresponding height profiles: (a) CCG, (b) BSA/CCG, (c) Chit/CCG, (d) DA/CCG, (e) DNA/CCG, (f) Gel/CCG and (g) PEG/CCG. All AFM topography images were collected using the heightsensor mode.

electrochemical biosensor system can be used for the highly sensitive detection of biomolecules. ${ }^{35,36}$ Here, we attempt to demonstrate the use of a graphene-based electrochemical biosensor in an array sensing application. In total, seven different graphene derivatives were used in the initial array (BSA/CCG, CCG, Chit/CCG, DA/CCG, DNA/CCG, Gel/CCG and PEG/CCG; Scheme 1b).

A physical characterization of the graphene derivatives was conducted prior to use. Fourier transform infrared spectra (Supplementary Figure S1) and thermogravimetric analysis results (Supplementary Figure S2) indicated that the biomolecule or synthetic polymer was successfully conjugated to the graphene. Transmission electron microscopy images (Supplementary Figure S3) revealed that all the samples dispersed separately with a thin, wrinkling, paper-like structure. Atomic force microscopy analysis (Figure $1 \mathrm{~b}-\mathrm{g}$ ) showed the height increase of BSA/CCG, Chit/CCG, DA/CCG, DNA/CCG, Gel/CCG and PEG/CCG nanosheets compared to pristine CCG $(<1.0 \mathrm{~nm}$, Figure 1a). As is widely known, GO is a nonconductive material due to the presence of oxygen functional groups located at its basal planes. Reduction of the oxygen functional groups in the synthesized graphene derivatives was confirmed by ultraviolet-visible spectra (Supplementary Figure S4) and X-ray photoelectron spectroscopy (Supplementary Figure S5). Zeta potential measurements provided information on the nanoparticle surface charges. As shown in Supplementary Figure S6, Chit/CCG is positively charged, while the other probes are more or less negatively charged in neutral PBS solution. The sensor array was generated by modifying the graphene nanosheets on a GCE. These modified electrodes were then incubated with the cells for $30 \mathrm{~min}$ to determine the changes in the electrochemical impedance of the electrodes. We first measured the electrochemical impedance of the constructed sensor array and used this impedance value as the baseline value for subsequent analyses (Supplementary Figure S7).

\section{Detection of differences in cancerous cell types}

For the initial discrimination study, the following five different types of human cancerous cells were tested: A549 (lung), HeLa (cervical), HepG2 (liver), K562 (leukemia) and MCF-7 (breast). Representative data arising from exposure of the sensor array to the cancerous cells are shown in Figure 2a and b and Supplementary Figure S8, as both 
a

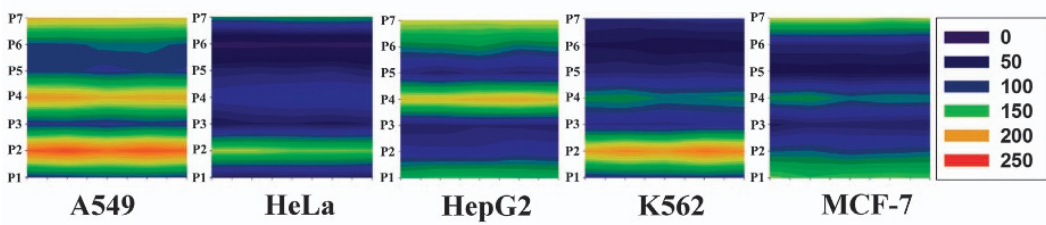

c

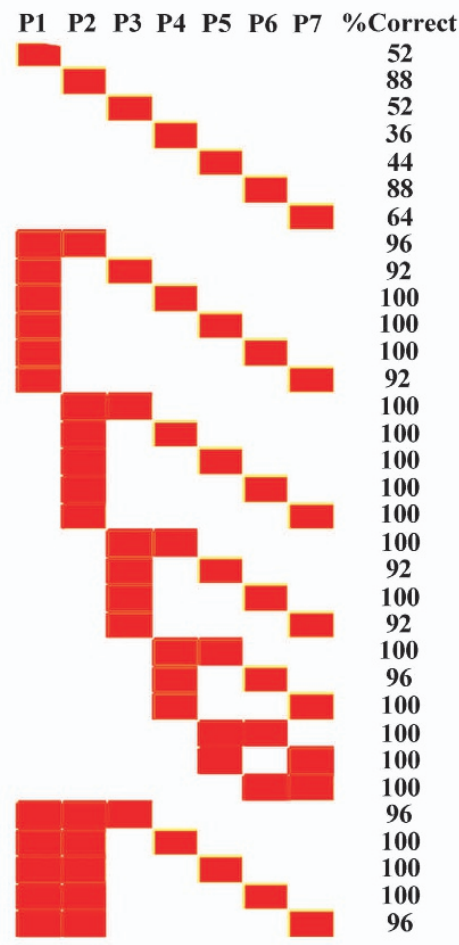

f b

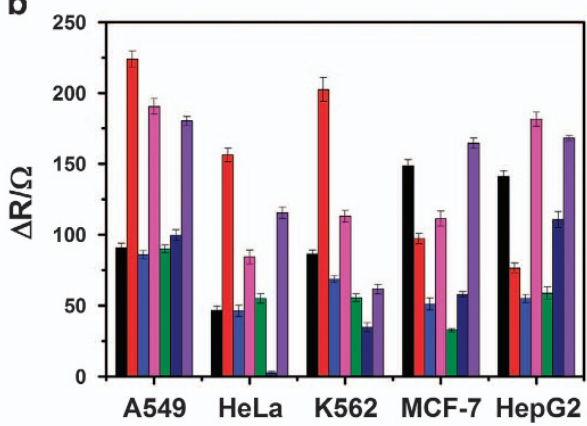

e
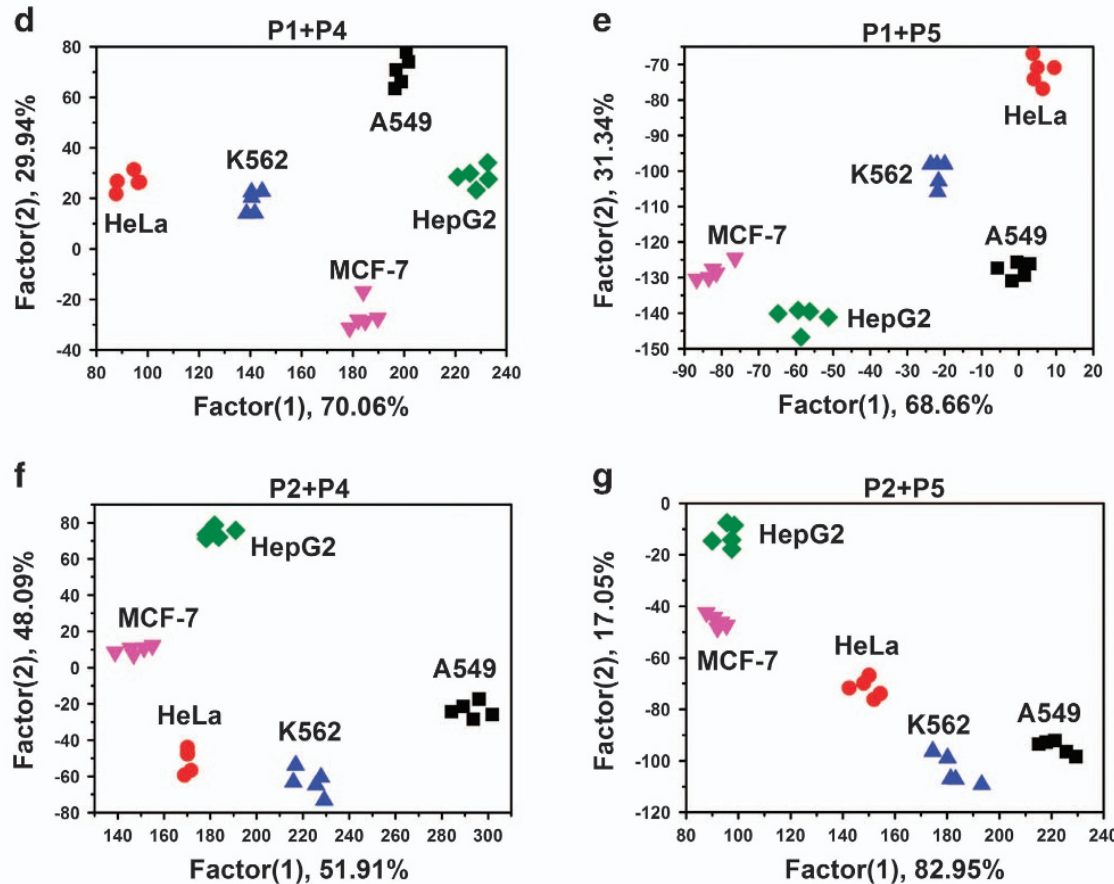

g

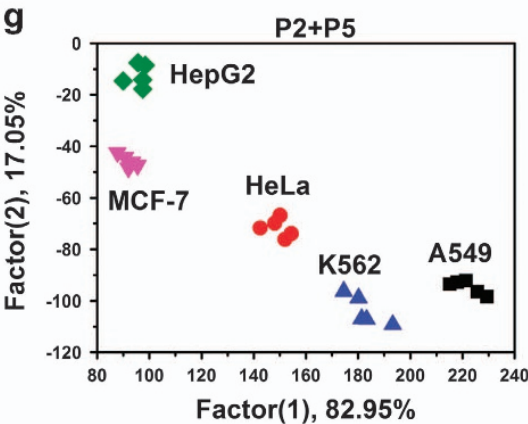

Figure 2 Differentiation of cancerous cell types based on the cell surface at a cell density of 100 cells: (a) 2D Electrochemistry contour plots of the cancerous cell response of the functionalized graphene probes (P1: BSA/CCG; P2: CCG; P3: Chit/CCG; P4: DA/CCG; P5: DNA/CCG, P6: Gel/CCG; and P7: PEG/CCG); (b) changes in $R_{\text {et }}$ (the electron-transfer resistance at the electrolyte/film interface) recorded by electrochemical impedance spectra (mean of five replicates) for five different cancerous cell lines using seven functionalized graphene probes; (c) Jackknifed classification matrix obtained using LDA for P1-P7 for five cancerous cell lines: A549, HeLa, HepG2, K562 and MCF-7; Canonical score plot for the sensor array containing P1 and P4 (d), P1 and P5 (e), P2 and P4 (f), and P2 and P5 (g).

2D electrochemistry contour plots and histograms. As illustrated in Figure 2a, each type of cell had its own individual color-change fingerprint. To deconvolute the electrochemical responses that provided discriminating signatures, we classified the electrochemical data set for all seven graphene probes using LDA. This statistical analysis approach was used to identify the linear combination of characteristics that discriminated multiple classes of objects. Jackknifed classification (stepwise analysis with various probe set(s)) was used to identify the probe set that could best discriminate the cancerous cells (Figure 2c). Jackknifed classification represents a typical cross-validation process of multiple discriminant analysis, in which cases are classified without using the misclassified individuals when calculating the classification function. This analysis reduced the size of the training matrix (2 graphene probes $/ 5$ cell types $/ 5$ replicates) and converted the matrix into canonical factors, which were linear combinations of the response matrices ( 2 factors $/ 5$ cell types/5 replicates). In the canonical electrochemical response patterns, each point represented the response pattern for a single-cell type to the graphene senor array and the different cell types were clustered into five nonoverlapping groups (Figure $2 \mathrm{~d}-\mathrm{g}$ and Supplementary Figure S9), indicating the ability of these sets of probes to differentiate between the five different cancer cell types using a simple graphene array. Previous array sensors typically required complex probe combinations (usually more than three probes) and achieved modest success with only a few of these probe sets attaining 100\% accuracy. The graphene-based electrochemical sensing platform presented here can more generally be applied to array sensor fabrication as it provided highly efficient signal transduction pathways and improved the detection response of each recognition element.

The robustness of the graphene-based array system was investigated with unknowns produced randomly by the five cancerous cell lines. The electrochemical impedance response patterns (Supplementary Table S1) were analyzed by LDA, and the cell types were classified in accordance with their Mahalanobis distances to the groups 


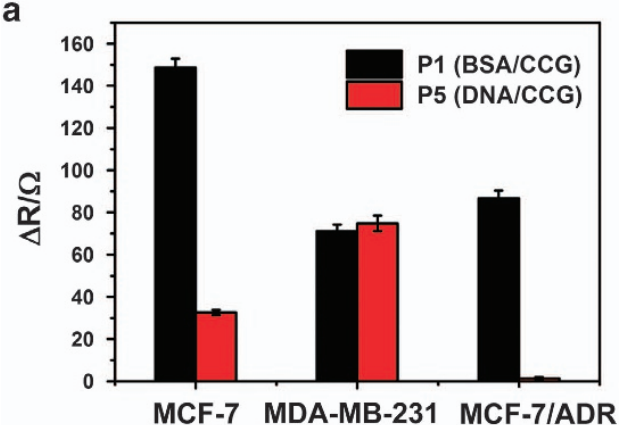

b

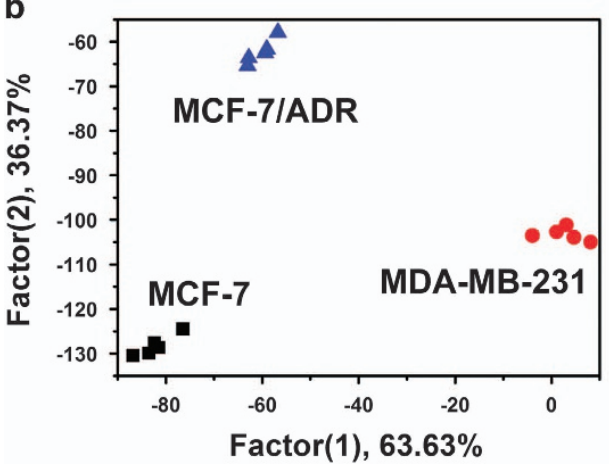

Figure 3 Detection of cancerous, multidrug-resistant and metastatic human breast cells at a cell density of 100 cells. (a) Change in the value of electrochemical impedance $\left(R-R_{0}\right)$ for 3 breast cell lines of different natures, MCF-7 (cancer), MCF-7/ADR (multidrug-resistant) and MDA-MB-231 (metastatic), using the ensemble of P1 and P5. Each value is the average of 5 parallel measurements. (b) Canonical score plot for the electrochemical response patterns obtained with the functionalized graphene assembly arrays against different mammalian cell types.

generated by the training matrix. In our study, the unknown samples were classified with $96.7 \%$ accuracy (29 out of 30 ).

\section{Detection of cancerous, multidrug-resistant cancerous and metastatic human breast cells}

Metastasis, in which some cancer cells are shed from the origin tumor and become rooted in different tissues, is the main cause of death from cancer. To date, the main treatment for many metastatic cancers is chemotherapy. However, multidrug resistance usually develops eventually; as a result, the use of chemotherapeutic drugs to kill cancer cells becomes less effective, causing the chemotherapeutic treatment to fail. As a consequence, multidrug resistance is one of the main barriers for the successful chemotherapeutic treatment of cancer. We chose three different human breast cell lines to test our sensor array in this application: MCF-7, a cancerous breast cell line; MDA-MB-231, a metastatic cancer cell line; and MCF-7/ADR, a multidrug-resistant cancer cell line. The three cell lines show differential electrochemical impedance patterns (Figure 3a). The two canonical factors contain 63.63 and $36.37 \%$ of the variation, as shown in Figure 3b. In the canonical electrochemical response patterns, the different cell types are clustered into 3 nonoverlapping groups, indicating that the sensor array combining P1 and P5 could differentiate cancer cell types phenotypically based on their surface properties.

Clinical cancer diagnosis applications require both the qualitative and quantitative identification of cancer cells. As shown in Figure 4a, varying cancer cell concentrations led to the drastic alteration of
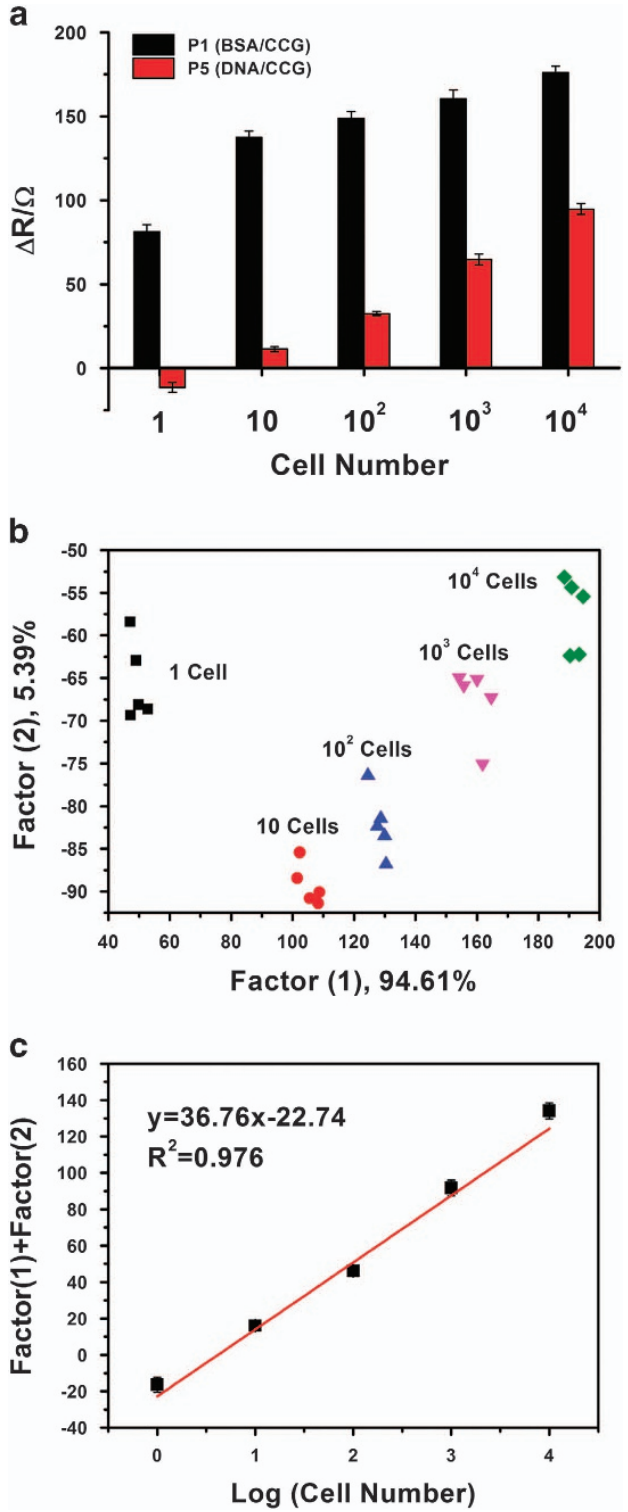

Figure 4 Identification of cancer cells at various concentrations using the sensor array consisting of $P 1$ and P5. (a) Changes in the value of electrochemical impedance $\left(R-R_{0}\right)$ for MCF-7 cells with different concentrations using the ensemble of P1 and P5. Each value is the average of 5 parallel measurements. (b) Canonical score plot for the electrochemical response patterns obtained with functionalized graphene assembly arrays against cancer cells at various concentrations. (c) Plot of the discriminant factor vs the logarithm of the MCF-7 concentration.

electrochemical response patterns for the cells. The graphene-based electrochemical sensor array fabricated here was sufficiently sensitive to identify cancer cells at the single-cell level (Figure $4 \mathrm{~b}$ and c). These results indicated that the sensing strategy had high efficacy in discriminating different cancer cell types and cancer states, and an ultrahigh sensitivity to quantify a single cancer cell type.

Identification of normal and cancerous cells

To validate the detection efficiency of our selective array-based sensing strategy, we performed further tests to identify normal and cancerous cells. The ability to differentiate cancerous cells from normal ones 
a

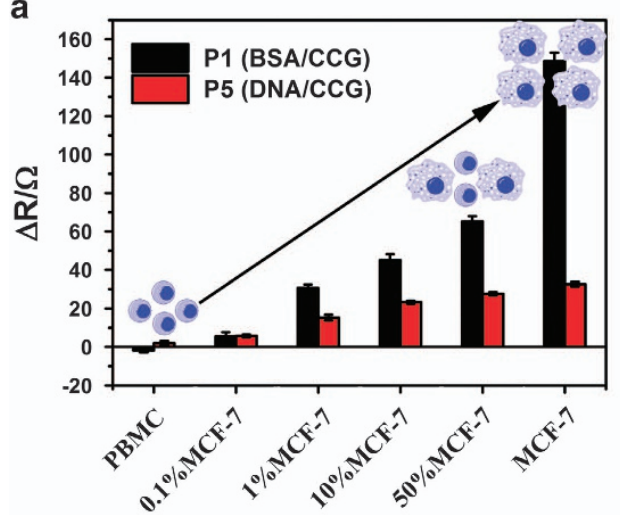

b

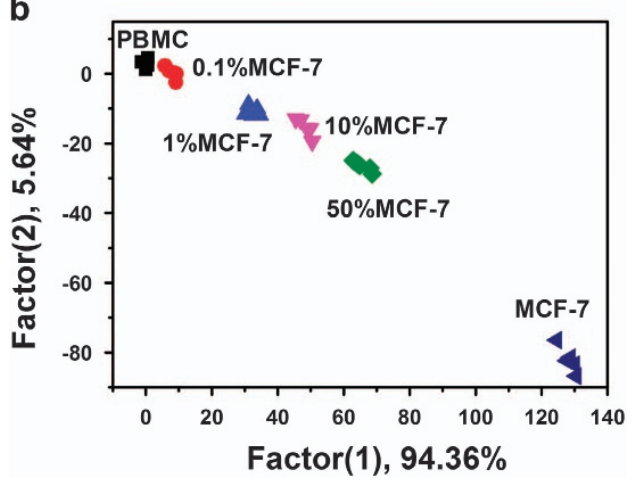

Figure 5 Identification of artificial CTC suspensions (prepared with a fixed total number of cells mixed by PBMC and MCF-7 cells in different cell ratios). (a) Changes in the value of electrochemical impedance $\left(R-R_{0}\right)$ for PBMC, artificial CTCs and MCF-7 cells using the ensemble of P1 and P5. Each value is the average of 5 parallel measurements. (b) Canonical score plot for the electrochemical response patterns obtained with the functionalized graphene assembly arrays against PBMC, artificial CTCs and MCF-7 cells.

represents a key requirement for diagnostic applications. The five cell lines (NIH-3T3, PC-12, HEK-293T, MCF-7 and A549) were screened with the seven graphene probes. Supplementary Figure S10 shows the electrochemical impedance patterns obtained from the seven functionalized graphene recognition elements after their incubation with the different cell types. The rational analysis of the above data represented by $2 \mathrm{D}$ electrochemistry contour plots (Supplementary Figure S11A) and histograms (Supplementary Figure S11B) showed that the electrochemical impedance enhancement of the array sensor reflected the original sensing information and could provide more subtle and accurate details. These patterns were reproducible and subjected to LDA to generate the jackknifed classification matrix. The jackknifed analysis (Supplementary Figure S12) indicated that maximum differentiation grouping was achieved using the two graphene probes. As shown in Supplementary Figure S13, 13 probe sets displayed 100\% identification accuracy among these cell types and LDA classified the cell types into five distinct clusters. Taken together, these studies indicated that our method could rapidly and effectively differentiate cell lines based on the noncovalent and reversible interactions between the cell surface components and the nanoparticle-based sensor elements.

\section{Detection of circulating tumor cells}

The development of simple and noninvasive cancer biomarker tests can classify tumors and allow early cancer detection. Thus, the most

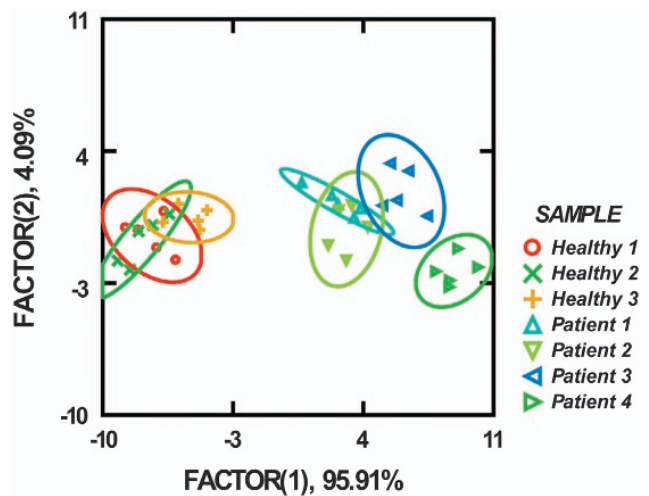

Figure 6 Canonical score plot for the electrochemical response patterns obtained with functionalized graphene assembly arrays against clinical blood samples collected from three healthy donors and four cancer patients.

appropriate therapy can be provided to the patient, and meanwhile, the disease progression, regression and recurrence can be monitored. ${ }^{3}$ CTCs, the cells circulating in the bloodstream that are shed from a primary tumor or metastasis into the vasculature, can reflect the molecular characteristics of cells within inner tumor masses. As a 'liquid biopsy', the quantification of CTCs can evaluate cancer dissemination, predict the prognosis and assess the therapeutic efficacy. ${ }^{37}$ At present, the Cell-Search assay approved by the FDA is an expensive and inefficient way of capturing CTCs, and a great deal of white blood cells often contaminate the enriched CTCs. Thus, the value of CTCs in diagnostics is not fully utilized. Over the past few decades, diverse detection methods for CTCs have been developed to alleviate the shortcomings of the immunomagnetic-separation-based Cell-Search assay. ${ }^{38,39}$ Unlike the existing CTC diagnosis approaches, here for the first time, the overall signature of CTCs was taken into account using array-based sensing approaches, and we attempted to identify CTCs without the antibody-dependent isolation procedures.

To probe the ability of our system in this application, we performed spiking experiments with PBMCs and MCF-7 cells at a fixed total number of cells with different cell ratios. We created a training matrix (two graphene probes $\times$ six samples $\times$ five replicates) with functionalized graphene and each of the artificial CTC samples. Each of the samples generated distinct electrochemical responses, as shown in Figure 5a. We found that the LDA plots for these artificial CTC samples were not random, but rather followed certain patterns, and could be differentiated from each other, with two canonical factors containing 94.36 and $5.64 \%$ of the variance (Figure 5b). We further spiked MCF-7 cells into whole blood and created a training matrix (Supplementary Table S7). As shown in Supplementary Figure S14, LDA also separated these samples into six distinct clusters. Encouraged by these promising results, we further applied the arraybased sensor for whole-blood analysis. The clinical whole-blood samples were collected from three healthy donors and four breast cancer patients. The breast cancer samples we obtained for our study were classified based on the stage of the cancer (1-4). The four patients were classified as follows (Supplementary Table S8): patient 1: stage 1 ; patient 2 : stage 2 ; patient 3 : stage 3 ; and patient 4 : stage 4 . As demonstrated in Figure 6, the simulated clusters, obtained by the same sensor array used earlier for the spiking experiments, were well confined, with no overlaps occurring between the healthy blood samples and breast cancer blood samples. Furthermore, the simulated 
clusters resulting from healthy blood overlapped, indicating that these samples had similar physicochemical properties. The simulated clusters resulting from the four breast cancer blood samples were close to each other without serious overlap, possibly due to the change of CTC numbers or phenotype during cancer evolution. ${ }^{12}$ All these results unambiguously indicate that our sensor platform holds substantial promise for CTC discrimination. Therefore, the graphene-based sensor array provides new opportunities for clinical cancer diagnosis by creating a global signature pattern of CTCs.

\section{CONCLUSION}

We fabricated a functionalized graphene-based electrochemical sensor array and demonstrated its utility in cell sensing. Based on this sensor array, different cancerous, multidrug-resistant cancerous and metastatic human breast cells, as well as artificial CTC samples were differentiated. The biomimetic interactions between the sensor array and the cell surface and an ultrasensitive electrochemical strategy provided a high-efficiency binding event-signal transduction platform, which was instrumental in improving the sensing performance. Remarkably, full differentiation was achieved to discern different cell types at levels as low as 100 cells by using two graphene recognition elements, and the detection limit of cancerous cells reached the single-cell level. Furthermore, the majority of probe sets with two combined graphene probes achieved 100\% classification accuracy, indicating that a simple sensor array had ample diagnostic capacity. The graphene-based electrochemical sensing platform presented here can more generally be applied to array sensor fabrication, representing a new method for future applications in clinical cancer diagnostics.

\section{CONFLICT OF INTEREST}

The authors declare no conflict of interest.

\section{ACKNOWLEDGEMENTS}

This work was supported by 973 Project (2012CB720602) and NSFC

(21210002, 21431007 and 21533008).

1 Etzioni, R., Urban, N., Ramsey, S., McIntosh, M., Schwartz, S., Reid, B., Radich, J., Anderson, G. \& Hartwell, L. The case for early detection. Nat. Rev. Cancer 3, 243-252 (2003).

2 Jemal, A., Center, M. M., DeSantis, C. \& Ward, E. M. Global patterns of cancer incidence and mortality rates and trends. Cancer Epidemiol Biomarkers Prev. 19, 1893-1907 (2010).

$3 \mathrm{Wu}, \mathrm{L}$. \& Qu, X. Cancer biomarker detection: recent achievements and challenges. Chem. Soc. Rev. 44, 2963-2997 (2015).

4 Duan, R., Zuo, X., Wang, S., Quan, X., Chen, D., Chen, Z., Jiang, L., Fan, C. \& Xia, F. Quadratic isothermal amplification for the detection of microRNA. Nat. Protoc. 9 597-607 (2014)

5 de la Rica, R. \& Stevens, M. M. Plasmonic ELISA for the ultrasensitive detection of disease biomarkers with the naked eye. Nat. Nanotechnol. 7, 821-824 (2012).

6 Krishnan, S., Mani, V., Wasalathanthri, D., Kumar, C. V. \& Rusling, J. F. Attomolar detection of a cancer biomarker protein in serum by surface plasmon resonance using superparamagnetic particle labels. Angew. Chem. Int. Ed. 50, 1175-1178 (2011).

7 Wu, G. H., Datar, R., Hansen, K., Thundat, T., Cote, R. \& Majumdar, A. Bioassay of prostate-specific antigen (PSA) using microcantilevers. Nat. Biotechnol. 19, 856-860 (2001).

8 Wang, Y., Zhou, K., Huang, G., Hensley, C., Huang, X., Ma, X., Zhao, T., Sumer, B., DeBerardinis, R. \& Gao, J. A nanoparticle-based strategy for the imaging of a broad range of tumours by nonlinear amplification of microenvironment signals. Nat. Mater. 13, 204-212 (2014)

9 Xiong, K., Wei, W., Jin, Y., Wang, S., Zhao, D., Wang, S., Gao, X., Qiao, C., Yue, H., Ma, G. \& Xie, H. Biomimetic immuno-magnetosomes for high-performance enrichment of circulating tumor cells. Adv. Mater. 28, 7929-7935 (2016).

10 Joosse, S. A. \& Pantel, K. Biologic challenges in the detection of circulating tumor cells. Cancer Res. 73, 8-11 (2013).
11 Ben, F. D., Turetta, M., Celetti, G., Piruska, A., Bulfoni, M., Cesselli, D., Huck, W. \& Scoles, G. A method for detecting circulating tumor cells based on the measurement of single-cell metabolism in droplet-based microfluidics. Angew. Chem. Int. Ed. 55, 1-5 (2016).

12 Johnson, E. S., Anand, R. K. \& Chiu, D. T. Improved detection by ensemble-decision aliquot ranking of circulating tumor cells with low numbers of a targeted surface antigen. Anal. Chem. 87, 9389-9395 (2015).

13 Askim, J. R., Mahmoudi, M. \& Suslick, K. S. Optical sensor arrays for chemical sensing: the optoelectronic nose. Chem. Soc. Rev. 42, 8649-8682 (2013).

14 Lavigne, J. J. \& Anslyn, E. V. Sensing a paradigm shift in the field of molecular recognition: from selective to differential receptors. Angew. Chem. Ind. Et 40, 3118-3130 (2001).

15 Persaud, K. \& Dodd, G. Analysis of discrimination mechanisms in the mammalian olfactory system using a model nose. Nature 299, 352-355 (1982).

16 Peng, G., Tisch, U., Adams, O., Hakim, M., Shehada, N., Broza, Y., Billan, S., Abdah-Bortnyak, R., Abraham Kuten, A. \& Haick, H. Diagnosing lung cancer in exhaled breath using gold nanoparticles. Nat. Nanotechnol. 4, 669-673 (2009).

17 Huang, Y., Li, F., Qin, M., Jiang, L. \& Song, Y. A Multi-stopband photonic-crystal microchip for high-performance metal-ion recognition based on fluorescent detection. Angew. Chem. Int. Ed. 52, 7296-7299 (2013).

18 Folmer-Andersen, J. F., Kitamura, M. \& Anslyn, E. V. Pattern-based discrimination of enantiomeric and structurally similar amino acids: an optical mimic of the mammalian taste response. J. Am. Chem. Soc. 128, 5652-5653 (2006).

19 Schiller, A., Wessling, R. A. \& Singaram, B. A fluorescent sensor array for saccharides based on boronic acid appended bipyridinium salts. Angew. Chem. Int. Ed. 46, 6457-6459 (2007).

20 De, M., Rana, S., Akpinar, H., Miranda, O., Arvizo, R., Bunz, U. \& Rotello, V. Sensing of proteins in human serum using conjugates of nanoparticles and green fluorescent protein. Nat. Chem. 1, 461-465 (2009).

21 Miranda, O. R., Li, X., Garcia-Gonzalez, L., Zhu, Z., Yan, B., Bunz, U. \& Rotello, V. Colorimetric bacteria sensing using a supramolecular enzyme-nanoparticle biosensor. J. Am. Chem. Soc. 133, 9650-9653 (2011).

22 Bajaj, A., Mirandaa, O., Kim, I., Phillips, R., Jerry, D., Bunz, U. \& Rotello, V. Detection and differentiation of normal, cancerous, and metastatic cells using nanoparticle-polymer sensor arrays. Proc. Natl Acad. Sci. USA 106, 10912-10916 (2009).

23 Hummers, W. S. \& Offeman, R. E. Preparation of graphitic oxide. J. Am. Chem. Soc. 80, 1339-1339 (1958).

24 Li, D., Muller, M. B., Gilje, S., Kaner, R. B. \& Wallace, G. G. Processable aqueous dispersions of graphene nanosheets. Nat. Nanotechnol. 3, 101-105 (2008).

25 Liu, J., Fu, S., Yuan, B., Li, Y. \& Deng, Z. Toward a universal 'adhesive nanosheet' for the assembly of multiple nanoparticles based on a protein-induced reduction/decoration of graphene oxide. J. Am. Chem. Soc. 132, 7279-7281 (2010).

26 Fang, M., Long, J., Zhao, W., Wang, L. \& Chen, G. pH-responsive chitosan-mediated graphene dispersions. Langmuir 26, 16771-16774 (2010).

27 Xu, L. Q., Yang, W. J., Neoh, K.-G., Kang, E.-T. \& Fu, G. D. Dopamine-induced reduction and functionalization of graphene oxide nanosheets. Macromolecules 43 , 8336-8339 (2010)

28 Qu, K., Wu, L., Ren, J. \& Qu, X. Natural DNA-modified graphene/Pd nanoparticles as highly active catalyst for formic acid electro-oxidation and for the suzuki reaction. ACS Appl. Mater. Interfaces 4, 5001-5009 (2012).

29 Liu, K., Zhang, J., Cheng, F., Zheng, T., Wang, C. \& Zhu, J. Green and facile synthesis of highly biocompatible graphene nanosheets and its application for cellular imaging and drug delivery. J. Mater. Chem. 21, 12034-12040 (2011).

30 Banerjee, S. S., Jalota-Badhwar, A., Satavalekar, S., Bhansali, S., Aher, N., Mascarenhas, R., Paul, D., Sharma, S. \& Khandare, J. Cell targeting: transferrinmediated rapid targeting, isolation, and detection of circulating tumor cells by multifunctional magneto-dendritic nanosystem. Adv. Healthc. Mater. 2. 770-770 (2013)

31 Wei, W. \& Qu, X. Extraordinary Physical properties of functionalized graphene. Small 8, 2138-2151 (2012).

32 Feng, L., Wu, L. \& Qu, X. New horizons for diagnostics and therapeutic applications of graphene and graphene oxide. Adv. Mater. 25, 168-186 (2013).

33 Pei, H., Li, J., Lv, M., Wang, J., Gao, J., Lu, J., Li, Y., Huang, Q., Hu, J. \& Fan, C. A graphene-based sensor array for high-precision and adaptive target identification with ensemble aptamers. J. Am. Chem. Soc. 134, 13843-13849 (2012).

34 Chou, S. S., De, M., Luo, J., Rotello, V., Huang, J. \& Dravid, V. Nanoscale graphene oxide (nGO) as artificial receptors: implications for biomolecular i nteractions and sensing. J. Am. Chem. Soc. 134, 16725-16733 (2012).

35 Feng, L., Wu, L., Wang, J., Ren, J., Miyoshi, D., Sugimoto, N. \& Qu, X. Detection of a prognostic indicator in early-stage cancer using functionalized graphene-based peptide sensors. Adv. Mater. 24, 125-131 (2012).

$36 \mathrm{Wu}$, L., Wang, J., Feng, L., Ren, J., Wei, W. \& Qu, X. Label-free ultrasensitive detection of human telomerase activity using porphyrin-functionalized graphene and electrochemiluminescence technique. Adv. Mater. 24, 2447-2452 (2012) 
37 Williams, S. C. P. Circulating tumor cells. Proc. Natl Acad. Sci. USA 110, 4861 (2013).

38 Fischer, J. C., Niederacherb, D., Topp, S. A., Honisch, E., Schumacher, S., Norma Schmitz, N., Föhrding, L., Vay, C., Hoffmann, I., Kasprowicz, N. S., Hepp, P. G., Mohrmann, S., Ulrike Nitz, U., Stresemann, A., Krahn, T., Henze, T., Griebsch, E., Raba, K., Rox, J. M., Wenzel, F., Sproll, C., Janni, W., Fehm, T., Klein, C. A., Knoefel, W. T. \& Stoecklein, N. H. Diagnostic Leukapheresis Enables Reliable detection of Circulating Tumor Cells of Nonmetastatic Cancer Patients. Proc. Natl. Acad. Sci. USA 110, 16580-16585 (2013).

39 Hou, S., Zhao, L., Shen, Q., Yu, J., Ng, C., Kong, X., Wu, D., Song, M., Shi, X., Xu, X., OuYang, W., He, R., Zhao, X., Lee, T., Brunicardi, F., Garcia, M., Ribas, A., Lo, R. \& Tseng, H. Polymer nanofiber-embedded microchips for detection, isolation, and molecular analysis of single circulating melanoma cells. Angew. Chem. Int. Ed. 52, 3379-3383 (2013). (c) (i) This work is licensed under a Creative Commons Attribution 4.0 International License. The images or other third party material in this article are included in the article's Creative Commons license, unless indicated otherwise in the credit line; if the material is not included under the Creative Commons license, users will need to obtain permission from the license holder to reproduce the material. To view a copy of this license, visit http:// creativecommons.org/licenses/by/4.0/

(C) The Author(s) 2017

Supplementary Information accompanies the paper on the NPG Asia Materials website (http://www.nature.com/am) 